doi:10.4149/neo_2017_510

\title{
Long non-coding RNA MALAT1 promotes cholangiocarcinoma cell proliferation and invasion by activating PI3K/Akt pathway
}

\author{
C. WANG ${ }^{1}$, Z. P. MAO ${ }^{1}$, L. WANG ${ }^{1}$, G. H. WU ${ }^{1}$, F. H. ZHANG ${ }^{1}$, D. Y. WANG ${ }^{1}$, J. L. SHI ${ }^{2, *}$ \\ ${ }^{1}$ Department of Oncology Surgery, the Affiliated Hospital of Logistics University of the Chinese People's Armed Police Forces (PAP), Tianjin, \\ China, 300162; ${ }^{2}$ Department of thoracic surgery, the Affiliated Heping Hospital of Changzhi Medical College, Changzhi, Shanxi, China, 046000
}

${ }^{*}$ Correspondence: dr_jialunshi@163.com

Received January 14, 2017 / Accepted March 8, 2017

\begin{abstract}
Increasing evidence indicated that metastasis-associated lung adenocarcinoma transcript 1 (MALAT1) acted as a key regulator in the proliferation and invasion of several cancers. However, the function of MALAT1 in the development of cholangiocarcinoma has not been experimentally established. In the present study, the expression levels of MALAT1 in cholangiocarcinoma cell lines were detected by quantitative real-time PCR. The effects of MALAT1 knockdown on the cell proliferation and invasion of cholangiocarcinoma cells were detected with Cell Counting Kit-8 (CCK-8), colony formation assay and Trans-well assay, respectively. The expressions of epithelial-mesenchymal transition (EMT)-related proteins (Ecadherin, Vimentin) were evaluated to discover whether the process of EMT was involved. We also evaluated the expression of phos-phatidylinositol-3-kinase/serine/threonine kinase (PI3K/Akt) signaling pathway proteins (PI3K, p-PI3K, Akt, p-Akt) to determine the associated molecular mechanism. And we discovered that MALAT1 was up-regulated in cholangiocarcinoma cancer cells. CCK-8, colony formation and trans-well assay showed that the proliferation and invasion of QBC-939 and RBE with MALAT1 knockdown were inhibited. Moreover, MALAT1 could promote EMT in cholangiocarcinoma cells. In addition, MALAT1 may activate PI3K/Akt pathway. These results indicated that MALAT1 promoted cholangiocarcinoma cell proliferation and invasion. The effects of MALAT1 on cholangiocarcinoma cells might be through activating the PI3K/ Akt signaling pathway. These investigations may facilitate a better understanding of MALAT1 and it might be a potential therapeutic target for the treatment of cholangiocarcinoma.
\end{abstract}

Key words: IncRNA, MALAT1, cholangiocarcinoma, EMT, PI3K/Akt pathway

Cholangiocarcinoma is a heterogeneous neoplasm that arises in diverse levels of the biliary duct, develops through carcinogenesis, and exhibits variable histologic morphologies and immunohistochemical profiles [1]. It is highly malignant and the incidence has increased over the recent decades. Unfortunately, we have not known much about cholangiocarcinoma at the present time. It is difficult to diagnose and assess the prognosis of patients with ICC in the clinic due to the lack of specific biomarkers. Surgical excision is considered as the only possible curative treatment for early stage patients with cholangiocarcinoma. A standardized or common therapeutic approach for this devastating disease is yet to be established.

Recently, promising evidence has shown that long noncoding RNAs (lncRNAs), that are more than 200 nucleotides in length, serve as ideal biomarkers and participate in cancer promotion and progression through involvement in key pathways. Currently, only a minority of lncRNAs have been reported to be associated with cholangiocarcinoma progression. Wang et al. [2] revealed that H19 and HULC, up-regulated by oxidative stress, regulate cholangiocarcinoma cell migration and invasion by targeting IL- 6 and CXCR4 via ceRNA patterns of sponging let-7a/let-7b and $\mathrm{miR}-372 / \mathrm{miR}-$ 373, respectively. A study from $\mathrm{Ma}$ et al. [3] demonstrated that the expression levels of Carbamoyl-phosphate synthase 1 (CPS1) and its lncRNA CPS1 intronic transcript 1 (CPS1-IT1) may serve a pivotal role in intrahepatic cholangiocarcinoma development by promoting the proliferation of cancer cells. Furthermore, the overexpression of CPS1 and CPS1-IT1 were associated with poor liver function and reduced survival rates, may represent potential biomarker and prognostic indicators for patients with intrahepatic cholangiocarcinoma. 
The metastasis-associated lung adenocarcinoma transcript 1 (MALAT1), also known as nuclear-enriched transcript 2 (NEAT2), is a nuclear-abundant long ncRNA and highly conserved in mammals [4]. MALAT1 was originally discovered in early stage non-small cell lung cancer and was identified as an independent prognostic marker for lung cancer metastasis [5], but also has been linked to several other human tumor entities [6-9]. Accumulating evidence indicated that MALAT1 was capable of effecting diverse cellular processes such as cell proliferation, apoptosis, cell-cycle progression, or cell growth, whether it has similar functions in cholangiocarcinoma has not been investigated.

Therefore, in this study, to investigate the role of MALAT1 in proliferation and invasion of cholangiocarcinoma, we detected the expression of MALAT1 in cholangiocarcinoma cells. Moreover, the expression levels of epithelial-mesenchymal transition (EMT)-related proteins (E-cadherin, Vimentin) were evaluated to discover whether the process of EMT was involved. We also evaluated the expression of phos-phatidylinositol-3-kinase/serine/threonine kinase (PI3K/Akt) signaling pathway proteins (PI3K, p-PI3K, Akt, p-Akt) to determine the associated molecular mechanism. These investigations may facilitate a better understanding of MALAT1 in cholangiocarcinoma.

\section{Materials and methods}

Cell culture. The three human cholangiocarcinoma cell lines used in this study were as follows: the HCCC-9810, QBC939 and RBE cell lines. The human normal biliary epithelial cell line H69 was used as the control. HCCC-9810, RBE cell lines were cultured in RPMI-1640 medium (Hyclone) with 10\% fetal bovine serum (FBS). QBC-939 and H69 cell lines were cultured in Dulbecco's modified Eagle's medium (DMEM) (Gibco) with $10 \%$ FBS. Cells were maintained at $37^{\circ} \mathrm{C}$ in a humidified incubator containing $5 \% \mathrm{CO}_{2}$. The medium was changed every three days.

Small interfering RNA transfection. QBC-939 and RBE cells were transfected with siRNA against MALAT1 to knock down MALAT1 using Lipofectamine 2000 transfection reagent (Invitrogen, CA, USA) according to the manufacturer's instructions. SiRNAs specifically targeting MALAT1 were designed and synthesized by Shanghai GenePharma Co, Ltd. SiRNA sequences for MALAT1: si1: 5'-GAGGUGUAAAGGGAUUUAUTT-3' (sense) and 5'-AUAAAUCCCUUUACACCUCTT-3' (antisense), si2: 5'-CACAGGGAAAGCGAGTGGTTGGTAA-3' (sense) and 5'-TTACCAACCACTCGCTTTCCCTGTG-3' (antisense). Negative control siRNA: 5'-GGCCUAAAGUAGUAGCUAUTT-3' (sense) and 5'-AUAGCUACUACUUUAGGCCTT-3' (antisense). Briefly, the cells in the logarithmic phase were seeded in 6-well plates before transfection. The confluence of cells reached $70-90 \%$ and serum-free medium were used for transfection. At $6 \mathrm{~h}$ after transfection, the medium was changed into the medium containing 10\% FBS. After $48 \mathrm{~h}$ of transfection, knockdown efficiency of MALAT1 was confirmed by real-time PCR.

RNA extraction, reverse transcription and qPCR. Total RNA was extracted using TRIZOL (TaKaRa, Dalian, China), according to the manufacturers' protocol. RNA was reversed transcribed into cDNA using the Primer-Script one step RT-PCR kit (TaKaRa, Dalian, China). The cDNA template was amplified by real-time RT-PCR using the SYBR Premix Dimmer Eraser kit (TaKaRa). Gene expression in each sample was normalized to GADPH expression. The primer sequences used were as follows: for GAPDH-forward, 5'-GTCAACGGATTTGGTCTGTATT-3' and GAPDH-reverse, 5'AGTCTTCTGGGTGGCAGTGAT-3', MALAT1-forward, 5'-ATGCGAGTTGTTCTCCGTCT-3' and MALAT1-reverse, 5'-TATCTGCGGTTTCCTCAAGC-3'. Real-time-PCR reactions were performed by the ABI7500 system (Applied Biosystems, Carlsbad, CA, USA). The qPCR amplification was performed in triplicate reactions beginning at $95^{\circ} \mathrm{C}$ for $10 \mathrm{~min}$, followed by 40 cycles of $95^{\circ} \mathrm{C}$ for $10 \mathrm{~s}$, and $60^{\circ} \mathrm{C}$ for 60 s. Quantitative normalization of MALAT1 cDNA was performed in each sample using the expression of the GAPDH as an internal control. The relative level of MALAT1 transcripts to control GAPDH was determined by the $2^{-\triangle \Delta C T}$ method.

Cell Counting Kit-8 (CCK-8) assay. Cell suspension was prepared, and the number of cells was counted using a cell counting board. Suspended cells with a density of $6000 / \mathrm{mL}$ were inoculated into a 96 -well plate $(200 \mu \mathrm{L}$ per well). When cells adhered to the wall, the culture medium was sucked out. After being washed with phosphate-buffered saline (PBS), DMEM or RPMI-1640 medium containing 10\% FBS was added for incubation $(200 \mu \mathrm{L}$ per well). Meanwhile, the same volume of medium was added to the control wells. After 24, $48,72 \mathrm{~h}$ of incubation at $37^{\circ} \mathrm{C}, 10 \mu \mathrm{L}$ of CCK- 8 was added to each well for $2 \mathrm{~h}$ of incubation. The absorbance value at 450 $\mathrm{nm}$ was measured by the enzyme-linked immunosorbent assay (ELISA) reader to calculate the proliferation rate and draw the related curve.

Colony formation assay. Colony formation assay was performed to assess the proliferation ability of QBC-939 and RBE cells. Cells in the logarithmic phase were trypsinised into a single-cell suspension $48 \mathrm{~h}$ after transfection. The cell suspension containing 500 cells were seeded in 6-well plate with the medium containing $10 \%$ FBS, and the plates were incubated in humidified atmosphere with $5 \% \mathrm{CO}_{2}$ at $37{ }^{\circ} \mathrm{C}$ for two weeks. Then the medium was removed and the plates were washed gently by phosphate buffered saline (PBS) twice, after which the cells were fixed by methanol for $15 \mathrm{~min}$ and stained in Giemsa (HarveyBio, Beijing, China) for $20 \mathrm{~min}$. After washed, the cells were air-dried, and colony number of each group was calculated. Colonies whose diameter was greater than $150 \mu \mathrm{m}$ were counted. Colony formation ratio was calculated as number of cells/initiative cell $\times 100(\%)$.

Trans-well invasion assay. Trans-well invasion assay was performed using Matrigel-coated (BD, Franklin Lakes, NJ, USA) filters in 24-well plates. Cells were trypsinized 
and seeded into the upper chambers of the trans-well $(1 \times$ $10^{5}$ cells/well) in serum-free DMEM medium. The lower chambers of the trans-well were filled with DMEM medium (including $10 \%$ fetal bovine serum). The chambers were incubated at $37^{\circ} \mathrm{C}$ and $5 \% \mathrm{CO}_{2}$ for $24 \mathrm{~h}$. At the end of incubation, cells on the upper surface of the filter were removed by wiping with a cotton swab. Cells migrating through the filter to the lower surface were fixed with $4 \%$ paraformaldehyde for $10 \mathrm{~min}$ and stained with $0.1 \%$ crystal violet for $10 \mathrm{~min}$. Cells washed 3 times in PBS were visualized using a phasecontrast microscope (Olympus, Tokyo, Japan) and counted from randomly chosen fields.

Western blot analysis. Cells were lysed in RIPA buffer containing fresh protease and phosphatase inhibitor cocktails (Sigma) by incubating for $15 \mathrm{~min}$ on the ice. Protein concentration were measured using the BCA assay (Beyotime Biotechnology, China) according to manufacturer's instructions. Whole protein extracts were resolved on a $10 \%$ SDS polyacrylamide gel and electrotransferred to polyvinylidene fluoride (PVDF) membranes (Millipore, US). The blot was blocked in 5\% skim milk for $2 \mathrm{~h}$ at room temperature and then incubated on a shaker overnight at $4^{\circ} \mathrm{C}$ in the specific primary antibodies. GAPDH (1:1000, Cell Signaling Technology) was used as an internal control. The primary antibodies used were anti-PCNA (1:1000, Santa Cruz, USA), anti-E-cadherin (1:500, Santa Cruz, USA), anti-Vimentin (1:500, Santa Cruz, USA), anti-PI3K (1:1000, Cell Signaling Technology), anti-Phospho-PI3K (1:1000, Cell Signaling Technology), anti-Akt (1:1000, Cell Signaling Technology), anti-Phospho-Akt (1:1000, Cell Signaling Technology). After washed in TBST (Tris Buffered Saline, with Tween-20) buffer, the blot was incubated in Horseradish peroxidase (HRP)conjugated secondary antibody (1:1000, Abcam) for $1 \mathrm{~h}$ at room temperature. Blots were developed using enhanced chemiluminescence detection reagents and scanned with a Molecular Imager system (Bio-Rad).

Statistical analysis. All statistical analyses were performed using SPSS 17.0 (SPSS, Chicago, IL). Experimental results were evaluated using the two-tailed Student $t$ test. All values were expressed as mean \pm SD. Statistical significance was noted at $P<0.05$. Three independent triplicated experiments were performed for cell biological assays, unless otherwise stated.

\section{Results}

The expression of MALAT1 was up-regulated in cholangiocarcinoma. To explore the relationship between MALAT1 and cholangiocarcinoma, we firstly explored the expression levels of MALAT1 in cholangiocarcinoma tissues in TCGA cholangiocarcinoma RNA-seq dataset (http:// syslab4.nchu.edu.tw/index.jsp), and there were 39 samples as follows: Stage 19, Stage II 10, Stage IVB 3 and Normal 7. As shown in Figure 1A, the levels of MALAT1 were significantly upregulated in Stage I and Stage cholangiocarcinoma patients. Although the overexpression of MALAT1 in Stage
B tissues was not significant, we considered that it was due to the short of the cholangiocarcinoma patients in Stage B. Then, we examined the expression of MALAT1 in cholangiocarcinoma cells HCCC-9810, RBE, QBC-939 and human normal biliary epithelial cell line H69. As shown in Figure $1 \mathrm{~B}$, the levels of MALAT1 were enhanced in cholangiocarcinoma cells compared to those in $\mathrm{H} 69$ cells. In addition, we

\section{A}

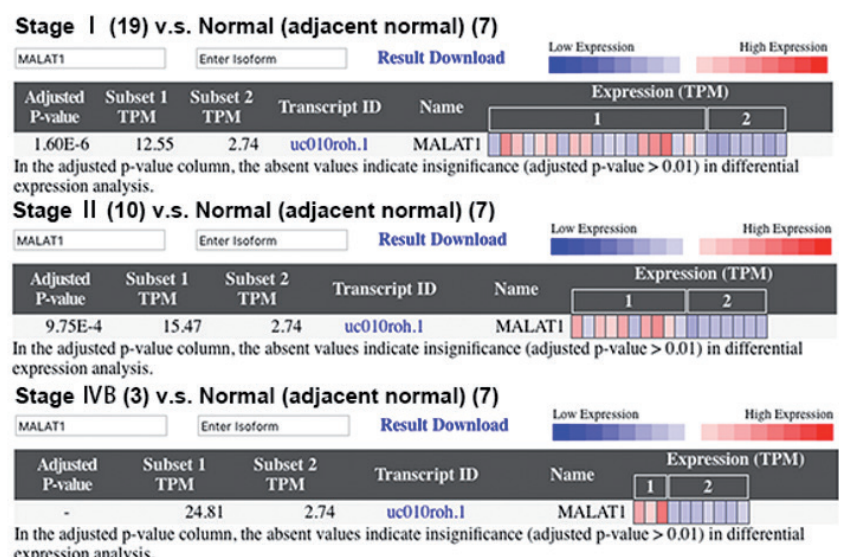

In the adjusted p-value column, the absent values indicate insignificance (adjusted p-value $>0.01$ ) in differenti-

B

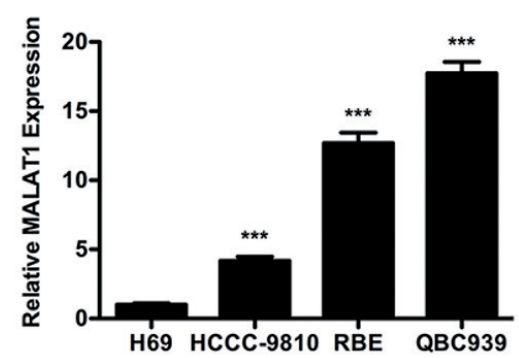

C

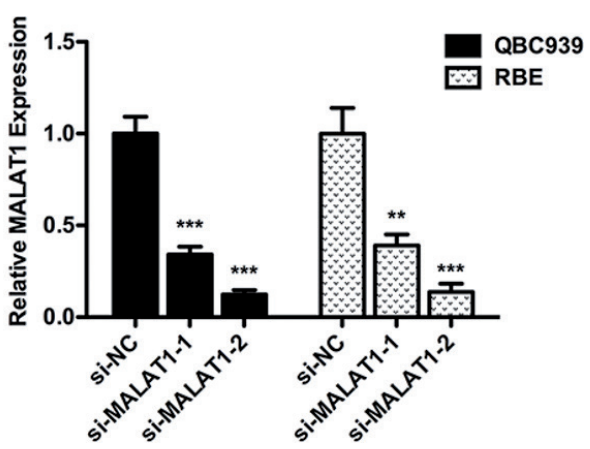

Figure 1. (A) The expression levels of MALAT1 in cholangiocarcinoma tissues in TCGA cholangiocarcinoma RNA-seq dataset. (B) Relative levels of Malat1 in normal $\mathrm{H} 69$ cells and three types of cholangiocarcinoma cells $\left({ }^{* *} P<0.001\right)$. (C) Efficacy of Malat1 interference in QBC-939 and RBE cells $\left({ }^{*} P<0.01,{ }^{* * *} P<0.001\right)$. qPCR amplification was performed in triplicate and the levels of MALAT1 were normalized against control GAPDH expression. 
chose QBC-939 and RBE cells that presented relatively high MALAT1 expression levels to knock down their expression of MALAT1 in the subsequent experiments. These data indicated that MALAT1 might be involved in the development of cholangiocarcinoma.

MALAT1 promoted the proliferation and invasion of cholangiocarcinoma cells. To investigate the role of MALAT1 in the proliferation and invasion of cholangiocarcinoma cells, cell viability and invasion ability were detected in QBC-939 and RBE cells transfected with si-MALAT1. According to the results of RT-qPCR, we chose si-MALAT1-2 to conduct further verification (Figure 1C).

Cell viability and proliferation were analyzed by CCK- 8 and colony formation assay, respectively. CCK-8 results showed that the viability of si-MALAT-treated cells was significantly inhibited QBC-939 cell viability at 48 and $72 \mathrm{~h}$ and inhibited
A

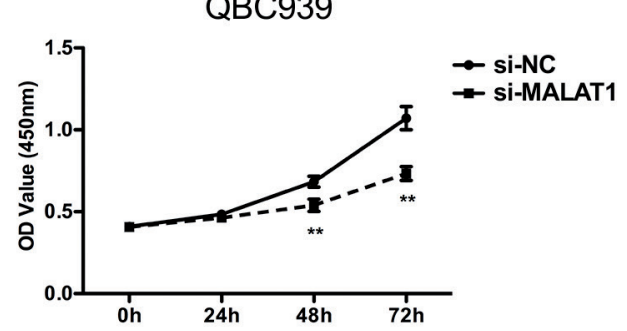

C

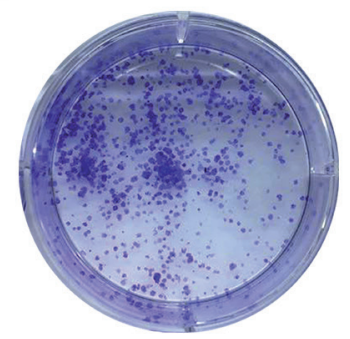

E

si-NC/QBC939

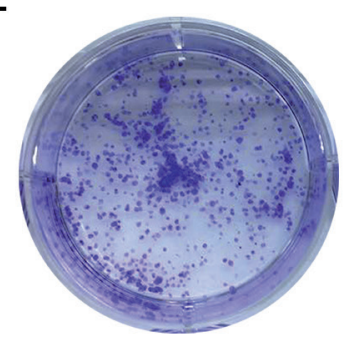

si-NC/RBE

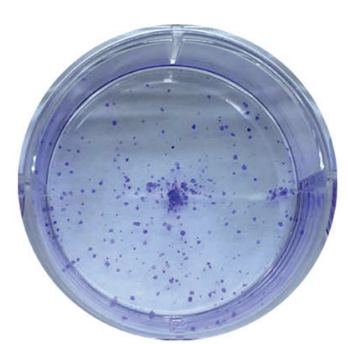

si-MALAT1/QBC939

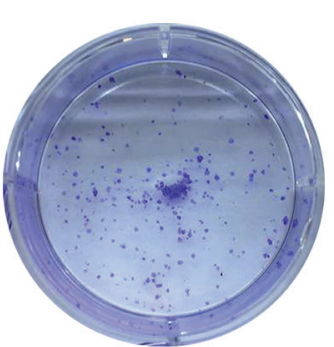

si-MALAT1/RBE

B

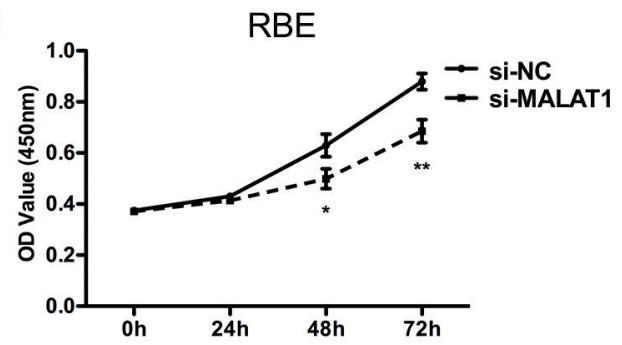

D
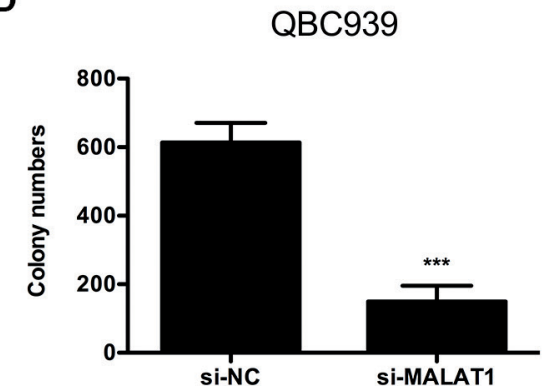

F

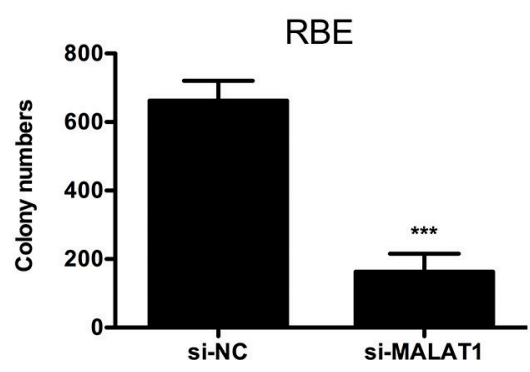

H

QBC939

\section{RBE}

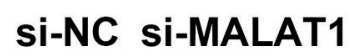

PCNA

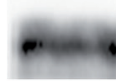

\section{PCNA}

\section{GAPDH}

\section{GAPDH}

Figure 2. MALAT1 promotes QBC-939 and RBE cells viability and proliferation. (A\&B) The cell viability of QBC-939 and RBE cells with MALAT1 knockdown. Cell viability was detected at $0,24,48,72 \mathrm{~h}$ after transfection at an optical density of $450 \mathrm{~nm}$. (C\&E) Pictures of colony formation assay of the four groups. Cells of si-MALAT1 groups formed fewer colonies than si-control when observed after two weeks of incubation. (D\&F) Histogram indicating the colony numbers of each group based on replicated colony formation assay $(* \star \star \quad P<0.001)$. (G\&H) The expression level of PCNA following MALAT1 knockdown. GAPDH is an internal control. PCNA, proliferating cell nuclear antigen. 
RBE cell viability at $72 \mathrm{~h}$ after transfection $(P<0.05$, Figure $2 \mathrm{~A}$ and $2 \mathrm{~B})$. Colony formation assay showed similar results: si-MALAT1 transfection reduced colony number compared to si-control (Figure 2C and 2E). Similar results were acquired in replicated experiments, with significant differences between groups $(P<0.001$, Figure $2 \mathrm{D}$ and $2 \mathrm{~F})$. Thus, these results indicated that knockdown of MALAT1 suppressed QBC-939 and RBE cells viability and proliferation. In addition, we detected the protein level of PCNA (Proliferating cell nuclear antigen), an endogenous marker for cell proliferation, by western blot. The Figures 2G and 2E demonstrated that the cell proliferation of cholangiocarcinoma cells treated with si-MALAT1 was suppressed.

Then we analyzed QBC-939 and RBE cell invasion using trans-well assay. Cell invasion results showed that si-MALAT1 transfection attenuated invasion ability more than si-control (Figure 3A and 3C). Similar results were acquired in replicated experiments, with significant differences between groups ( $P$ $<0.001$, Figure 3B and 3D). Taken together, knockdown of MALAT1 inhibited QBC-939 and RBE cells proliferation and invasion. These results suggest that increased expression of MALAT1 may be important for cholangiocarcinoma tumorigenesis and cancer progression.

MALAT1 promoted Epithelial-mesenchymal transition in cholangiocarcinoma cells. To further investigate the molecular mechanisms mediating the proliferation and invasion features of MALAT1, the protein expression levels of Epithelial-mesenchymal transition (EMT) markers were examined by western blotting assay. Results revealed that E-cadherin was significantly increased and Vimentin was reduced in si-MALAT1 cells compared to control cells (Figure $4 \mathrm{E}$ and $4 \mathrm{~F}$ ). Specifically, E-cadherin is the epithelial marker and Vimentin is the major cytoskeletal component of mesenchymal cells. Because of this, Vimentin is often used as
A

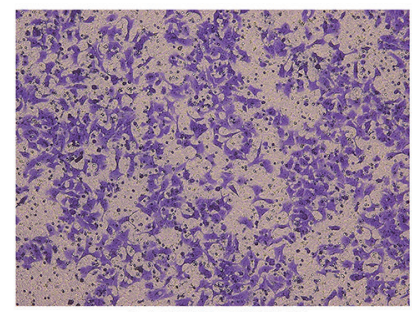

si-NC/QBC939

C

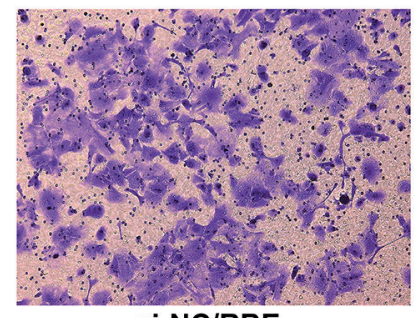

si-NC/RBE

E

QBC939

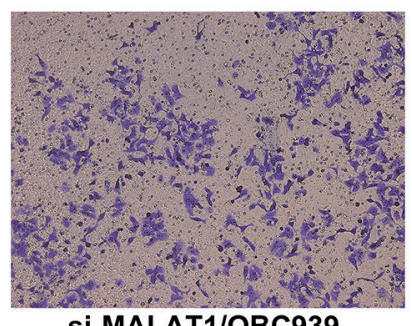

Si-MALAT1/QBC939

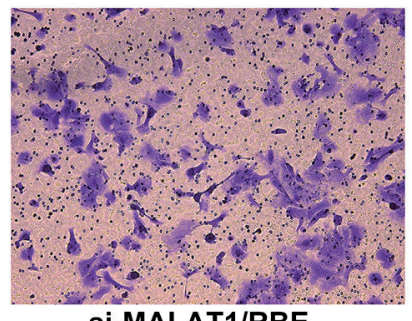

si-MALAT1/RBE

$\mathbf{F}$
B

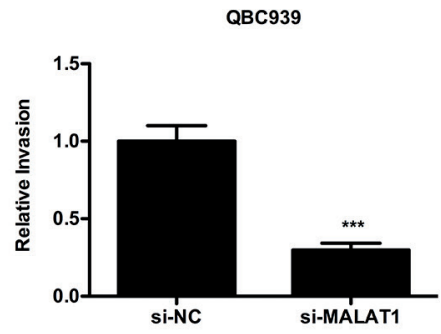

D

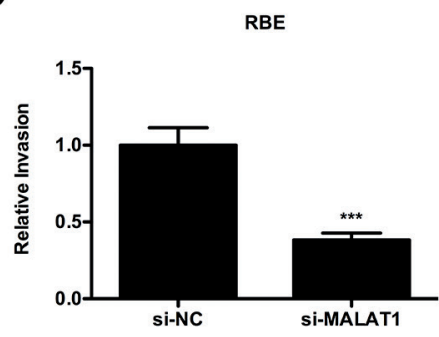

RBE
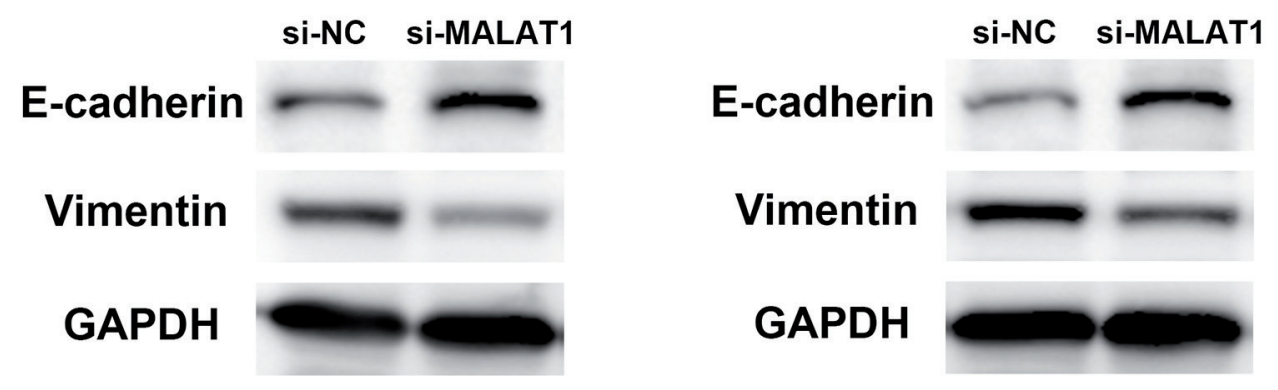

Figure 3. MALAT1 promotes cholangiocarcinoma cell invasion and EMT. (A\&C) Pictures of invasive cells detected by Trans-well assay with Matrigel at $48 \mathrm{~h}$ after MALAT1 knockdown vs si-control. (B\&D) Histogram of invasive cell number based on replicated experiments $\left({ }^{* *} P<0.001\right)$, results showed that the invasion of QBC-939 and RBE with MALAT1 knockdown were inhibited. (E\&F) The expression of EMT-related proteins (E-cadherin, Vimentin) was evaluated to discover whether the process of EMT was involved. Results revealed that E-cadherin was significantly increased and Vimentin was reduced in si-MALAT1 cells compared to control cells. 
QBC939

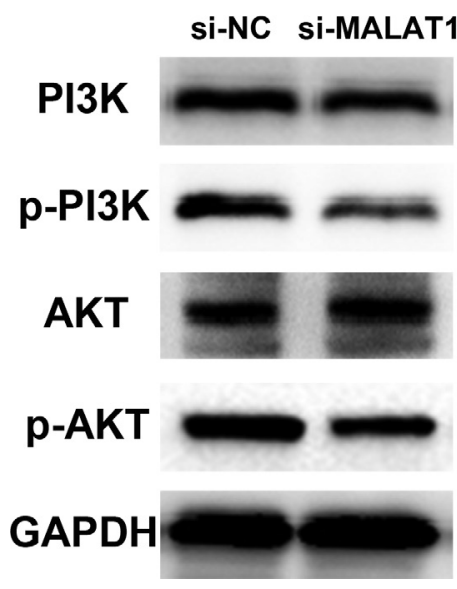

RBE si-NC SI-MALAT1

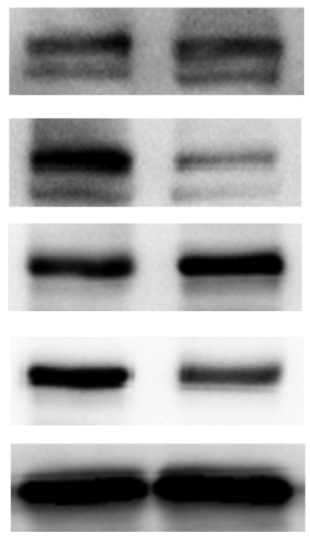

Figure 4. Protein levels of PI3K/Akt factors in cholangiocarcinoma cells. Western blot is performed at $48 \mathrm{~h}$ after transfection. GAPDH is an internal control. Levels of PI3K and Akt are hardly changed, while their phosphorylation forms p-PI3K and p-Akt are altered by si-MALAT1 transfection. The results demonstrated that the effects of MALAT1 on cholangiocarcinoma cells might be through activating the PI3K/Akt signaling pathway.

a marker of mesenchymally-derived cells or cells undergoing an EMT. These results demonstrated that MALAT1 promotes cholangiocarcinoma cell invasion through regulating EMTrelated proteins.

MALAT1 activated the PI3K/Akt pathway. Next we detected the expression of two factors in PI3K/Akt pathways and their phosphorylation forms by western blot to analyze the effects of MALAT1 on PI3K/Akt pathways. Results showed that the total protein level of PI3K, Akt were hardly changed by si-MALAT1 transfection in QBC-939 and RBE cells. However, the phosphorylation forms of the two proteins, namely, p-PI3K and p-Akt, were both down-regulated by si-MALAT1 compared to control groups (Figure 5). Based on these results, knockdown of MALAT1 was able to inhibit the activation of the two PI3K/Akt factors.

\section{Discussion}

Recently, Tan et al. [10] reported that MALAT1 was significantly upregulated in human hilar cholangiocarcinoma tissues and cell lines, and its oncogenic effect might through regulating CXCR4 via miR-204. In the present study, we profiled MALAT1 expression in human normal bile duct cell and cholangiocarcinoma cells, and identified MALAT1 was upregulated in cancer cells. CCK-8 assay, colony formation assay and trans-well invasion assay showed that the proliferation and invasion of QBC-939 and RBE cells with MALAT1 knockdown were inhibited. Moreover, the expression of EMT marker E-cadherin was highly increased, Vimentin was significantly reduced by si-MALAT1, which indicated that MALAT1 may promote invasion through regulating EMT in cholangiocarcinoma cells. In addition, MALAT1 could activate PI3K/Akt pathway. Taken together, our results suggest that MALAT1 promotes invasion of cholangiocarcinoma cells via regulating EMT and activating PI3K/Akt pathway.

MALAT-1 is a long ncRNA located on chromosome 11q13 and has been found to be associated with regulation of gene expressions. Increasing evidence proved that MALAT1 acted as a key regulator of the proliferation and invasion of several cancers including lung, breast, pancreas, liver, colon, uterus, cervix and prostate by targeting gene expression [11-13]. Whereas, there is no molecular target that has been obtained for cholangiocarcinoma diagnosis. Thus, exploring novel biomarkers especially epigenetic markers such as noncoding RNAs are urgently needed for early diagnosis of cholangiocarcinoma.

EMT has been found to be involved in the invasive fronts of human tumors. Thus, to further understand the underlying mechanism in cell invasion, we analyzed the protein expression of EMT. Our results showed that si-MALAT1 down-regulates the levels of Vimentin and up-regulates the levels of E-cadherin significantly. Vimentin is a marker positively related to the invasion and metastasis of tumor cells, whereas E-cadherin is a negative correlation marker [14]. Reduction or loss of E-cadherin is a well-established hallmark of EMT, and E-cadherin down-regulation is associated with a poor clinical prognosis for many cancers. Taken together, these results provided the evidence that MALAT1 promotes cholangiocarcinoma cell invasion through regulating EMT-related proteins.

$\mathrm{PI} 3 \mathrm{~K} / \mathrm{Akt}$ signaling pathway is aberrant in a wide variety of cancers and has been shown to play a central role in cancer cell proliferation, migration, invasion, and apoptosis [15-17]. Downstream effectors of AKT are involved in survival, growth and metabolic-related pathways [18]. The PI3K/ Akt signaling pathway is also responsible for modulating multiple processes in cholangiocarcinoma $[19,20]$. Thus, we detected the PI3K/Akt signaling pathway proteins to reveal the molecular mechanisms of MALAT1-knockdown cholangiocarcinoma cells. Our results showed that si-MALAT1 decreased the level of p-PI3K and p-Akt, but did not significantly decrease PI3K and Akt. The phosphorylations of PI3K and Akt were inhibited, indicating that MALAT1 may affect cholangiocarcinoma cells through suppressing the PI3K/Akt signaling pathway. This result is consistent with the findings of Dong et al. that MALAT1 promotes the proliferation and metastasis of osteosarcoma cells by activating the PI3K/Akt pathway [21].

\section{Conclusion}

In conclusion, we demonstrated that MALAT1 promoted cholangiocarcinoma cell proliferation and invasion. The effects of MALAT1 on cholangiocarcinoma cells might be through activating the PI3K/Akt signaling pathway. Mechanistically, 
MALAT1 may function as an inducer of EMT in cholangiocarcinoma by activating the PI3K/Akt pathway. MALAT1 might be a potential therapeutic target for the treatment of cholangiocarcinoma. However, further investigations should be performed to confirm this conclusion.

Acknowledgments: We thank to all the laboratory members for continuous technical advice and helpful discussion.

\section{References}

[1] EBATA T, ERCOLANI G, ALVARO D, RIBERO D, DI TOMMASO L et al. Current Status on Cholangiocarcinoma and Gallbladder Cancer. Liver Cancer 2016; 6: 59-65. https://doi. org/10.1159/000449493

[2] WANG WT, YE H, WEI PP, HAN BW, HE B et al. LncRNAs H19 and HULC, activated by oxidative stress, promote cell migration and invasion in cholangiocarcinoma through a ceRNA manner. J Hematol Oncol 2016; 9: 117. https://doi. org/10.1186/s13045-016-0348-0

[3] MA SL, LI AJ, HU ZY, SHANG FS, WU MC. Coexpression of the carbamoylphosphate synthase 1 gene and its long noncoding RNA correlates with poor prognosis of patients with intrahepatic cholangiocarcinoma. Mol Med Rep 2015; 12: 7915-7926. https://doi.org/10.3892/mmr.2015.4435

[4] GURU SC, AGARWAL SK, MANICKAM P, OLUFEMI SE, CRABTREE JS et al. A transcript map for the $2.8-\mathrm{Mb}$ region containing the multiple endocrine neoplasia type 1 locus. Genome Res 1997; 7: 725-735. https://doi.org/10.1101/gr.7.7.725

[5] JI P, DIEDERICHS S, WANG W, BOING S, METZGER R et al. MALAT-1, a novel noncoding RNA, and thymosin beta4 predict metastasis and survival in early-stage non-small cell lung cancer. Oncogene 2003; 22: 8031-8041. https://doi. org/10.1038/sj.onc. 1206928

[6] HU L, WU Y, TAN D, MENG H, WANG K et al. Up-regulation of long noncoding RNA MALAT1 contributes to proliferation and metastasis in esophageal squamous cell carcinoma. J Exp Clin Cancer Res 2015; 34: 7. https://doi.org/10.1186/s13046015-0123-Z

[7] MA KX, WANG HJ, LI XR, LI T, SU G et al. Long noncoding RNA MALAT1 associates with the malignant status and poor prognosis in glioma. Tumour Biol 2015; 36: 3355-3359. https://doi.org/10.1007/s13277-014-2969-7

[8] WU XS, WANG XA, WU WG, HU YP, LI ML et al. MALAT1 promotes the proliferation and metastasis of gallbladder cancer cells by activating the ERK/MAPK pathway. Cancer Biol Ther 2014; 15: 806-814. https://doi.org/10.4161/cbt.28584

[9] YING L, CHEN Q, WANG Y, ZHOU Z, HUANG Y et al. Upregulated MALAT-1 contributes to bladder cancer cell migration by inducing epithelial-to-mesenchymal transition. Mol Biosyst 2012; 8: 2289-2294. https://doi.org/10.1039/ c2mb25070e
[10] TAN X, HUANG Z, LI X. Long Non-Coding RNA MALAT1 Interacted with miR-204 to Modulates Human Hilar Cholangiocarcinoma Proliferation, Migration and Invasion by Targeting CXCR4. J Cell Biochem 2017. https://doi. org/10.1002/jcb.25862

[11] WU Y, LU W, XU J, SHI Y, ZHANG H et al. Prognostic value of long non-coding RNA MALAT1 in cancer patients. Tumour Biol 2016; 37: 897-903. https://doi.org/10.1007/s13277-015-3870-8

[12] GUTSCHNER T, HAMMERLE M, EISSMANN M, HSU J, KIM Y et al. The noncoding RNA MALAT1 is a critical regulator of the metastasis phenotype of lung cancer cells. Cancer Res 2013; 73: 1180-1189. https://doi.org/10.1158/0008-5472. CAN-12-2850

[13] SHI X, SUN M, LIU H, YAO Y, SONG Y. Long non-coding RNAs: a new frontier in the study of human diseases. Cancer Lett 2013; 339: 159-166. https://doi.org/10.1016/j. canlet.2013.06.013

[14] THIERY JP, ACLOQUE H, HUANG RY, NIETO MA. Epithelial-mesenchymal transitions in development and disease. Cell 2009; 139: 871-890. https://doi.org/10.1016/j.cell.2009.11.007

[15] MA LI, CHANG Y, YU L, HE W, LIU Y. Pro-apoptotic and anti-proliferative effects of mitofusin-2 via PI3K/Akt signaling in breast cancer cells. Oncol Lett 2015; 10: 3816-3822. https:// doi.org/10.3892/ol.2015.3748

[16] ZHANG FY, HU Y, QUE ZY, WANG P, LIU YH et al. Shikonin Inhibits the Migration and Invasion of Human Glioblastoma Cells by Targeting Phosphorylated beta-Catenin and Phosphorylated PI3K/Akt: A Potential Mechanism for the Anti-Glioma Efficacy of a Traditional Chinese Herbal Medicine. Int J Mol Sci 2015; 16: 23823-23848. https://doi. org/10.3390/ijms161023823

[17] YE Y, TANG X, SUN Z, CHEN S. Upregulated WDR26 serves as a scaffold to coordinate PI3K/ AKT pathway-driven breast cancer cell growth, migration, and invasion. Oncotarget 2016; 7: 17854-17869. https://doi.org/10.18632/oncotarget.7439

[18] ZHU QS, ROSENBLATT K, HUANG KL, LAHAT G, BROBEY R et al. Vimentin is a novel AKT1 target mediating motility and invasion. Oncogene 2011; 30: 457-470. https:// doi.org/10.1038/onc.2010.421

[19] RIZVI S, GORES GJ. Pathogenesis, diagnosis, and management of cholangiocarcinoma. Gastroenterology 2013; 145: 1215-1229. https://doi.org/10.1053/j.gastro.2013.10.013

[20] UTISPAN K, SONONGBUA J, THUWAJIT P, CHAU-IN S, PAIROJKUL C et al. Periostin activates integrin alpha5beta 1 through a PI3K/AKTdependent pathway in invasion of cholangiocarcinoma. Int J Oncol 2012; 41: 1110-1118. https://doi. org/10.3892/ijo.2012.1530

[21] DONG Y, LIANG G, YUAN B, YANG C, GAO R et al. MALAT1 promotes the proliferation and metastasis of osteosarcoma cells by activating the PI3K/Akt pathway. Tumour Biol 2015; 36: 1477-1486. https://doi.org/10.1007/s13277$\underline{014-2631-4}$ 